\title{
Distribución del Mono Nocturno Andino Aotus lemurinus en el departamento del Quindío, Colombia
}

\section{Sebastián O. Montilla}

Programa de Biología, Facultad de Ciencias básicas y Tecnologías, Universidad del Quindío, Grupo de Estudio en Mamíferos Silvestres (GEMS). juansomontilla94@gmail.com.

\section{Juan Camilo Cepeda-Duque}

Programa de Biología, Facultad de Ciencias Básicas, Universidad de Santa Rosa de Cabal, Grupo de Investigación en Biología de la Conservación y Biotecnología

\section{Sebastián Bustamante-Manrique}

Programa de Biología, Facultad de Ciencias Exactas y Naturales, Universidad de Caldas, Semillero de Investigación en Primatología y Conservación de sus Ecosistemas (SIPCE)

El Mono Nocturno Andino, Aotus lemurinus (Geoffroy 1846) (Figura 1A) es una especie de primate neotropical que se distribuye sobre los $1000 \mathrm{msnm}$ en la cordillera de los Andes de Ecuador, Colombia y el suroccidente de Venezuela (MoralesJiménez \& de la Torre 2008, Defler 2010). En Colombia, A. lemurinus ocurre en las dos vertientes de las tres cordilleras (Figura 1B), abarcando diferentes tipos de hábitats, que van desde bosques considerablemente conservados, hasta bosques secundarios, guaduales y plantaciones de café (Castaño \& Cardona 2005, Castaño et al. 2010, Defler 2010).
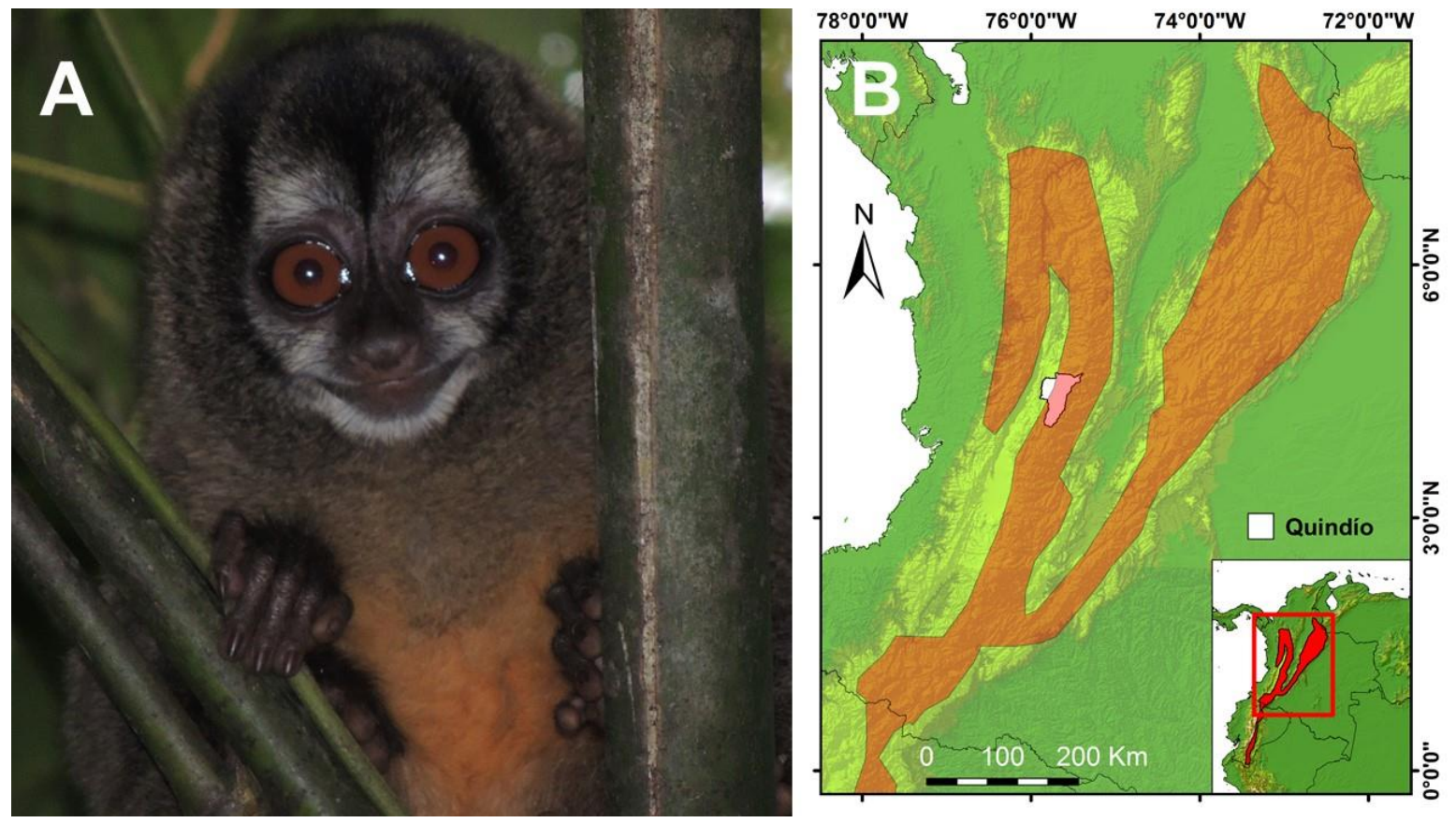

Figura 1. Mono nocturno andino (Aotus lemurinus) en la Finca El Billar, Pijao, Quindío (A). Mapa de distribución de A. lemurinus según la IUCN (Morales-Jiménez \& de la Torre 2008) (B). En blanco el departamento del Quindío

Actualmente la especie A. lemurinus se encuentra catalogada por la Unión Internacional para la Conservación de la Naturaleza (UICN) bajo la categoría de Vulnerable (VU) a la extinción, debido principalmente a la pérdida de hábitat producto de la deforestación y la expansión agrícola (Morales-Jiménez \& de la Torre 2008). En el eje cafetero las amenazas para A. lemurinus se han incrementado como resultado de las modificaciones en los cultivos de café, provocado por la eliminación de los cafetales con sombrío, generando a su vez perdida de conectividad entre los fragmentos de bosque (Borrero 1986). En la 
actualidad, se desconocen muchos aspectos importantes acerca de la ecología de A. lemurinus y los registros de esta especie son muy escasos, lo cual dificulta la implementación de medidas de manejo que aseguren su conservación a nivel regional. Por lo tanto, el presente trabajo tiene por objetivo aportar información sobre la distribución del Mono Nocturno Andino en el departamento del Quindío.

El departamento del Quindío se ubica en la vertiente occidental de la cordillera central de Colombia, desde los 950 hasta los $4750 \mathrm{msnm}$. Este departamento presenta diferentes tipos de ecosistemas, tales como bosque subandino, andino medio, altoandino, bosque secundario, bosque ripario, páramo, y guaduales, junto con una matriz mixta de agroecosistemas compuestos por policultivos y monocultivos donde predomina el café (Arbeláez-Cortés et al. 2011).

La presencia de A. lemurinus fue determinada a partir de observaciones ocasionales y búsquedas libres entre el año 2014 y 2016 en diferentes lugares del Quindío. Al momento de observar un individuo o grupo familiar, se georreferenció el lugar de observación y posteriormente se categorizó de acuerdo a la composición del paisaje donde se ubica el fragmento boscoso y el tamaño del mismo. Dichas características de cada bosque se determinaron a partir de imágenes satelitales extraídas del SigQuindío. Las categorías para cada bosque según la composición del paisaje fueron: 1. Bosque de vegetación continua; 2. Fragmento de bosque inmerso en cultivos; 3. Fragmento de bosque inmerso en potreros, y; 4. Fragmento de bosque periurbano. En cuanto al tamaño de los bosques, las categorías fueron 1. Menor a 5 ha; 2. Entre 5 y 50 hectáreas, y; 3. Mayor a 50 hectáreas. Adicionalmente se realizaron búsquedas de registros de A. lemurinus en el Quindío en diferentes bases de datos de biodiversidad (GBIF y SIB Colombia), bases de datos de museos (AMNH, FMNH, NMNH, ICN, MHNUC), artículos científicos de inventarios y trabajos relacionados con la especie. Por último, se realizó un mapa de distribución con las coordenadas de los registros propios del trabajo y los registros de literatura. Cabe mencionar que para los registros de literatura no se tuvo en cuenta ni la matriz del paisaje donde se encontraban los bosques ni su tamaño, debido a que estos registros no son recientes y se desconocen las características al momento del registro.

Respecto a la determinación taxonómica se consultó como material de referencia un individuo de A. lemurinus depositado en la Universidad de Caldas (MHNUC-687), y se siguieron las descripciones realizadas por Hernández-Camacho \& Cooper (1986) para la especie. En dichas descripciones se indica que A. lemurinus presenta dos tipos de coloraciones básicas en el dorso, gris castaño o castaño rojizo, ambas con el vientre amarillo y con el pelaje notablemente más largo que otras especies de Aotus. Además, por distribución se sugiere que la especie de mono nocturno propia de los Andes de Colombia, Ecuador y Venezuela es A. lemurinus (Defler 2010). Por lo tanto, no se incluyeron aquellos registros que estuviesen cercanos o por debajo de los 1000 msnm en el occidente del Quindío.

En total, a partir de las búsquedas libres se registró la presencia del mono nocturno andino en 13 localidades del departamento del Quindío (Tabla 1). Dichos registros se suman a cinco registros más obtenidos a partir de literatura (Castaño \& Cardona 2005) y especímenes depositados en el Museo de Historia Natural de la Universidad de Caldas y en el American Museum of Natural History (MHNUC-M00072, MHNU-687, MHNUC-901, y M-33056). Todos los registros se dieron en la porción cordillerana del departamento del Quindío, cubriendo ocho de los 12 municipios que lo componen (Figura 2). Se presentaron registros desde los $1250 \mathrm{msnm}$, en la finca Paraguaicito en el municipio de Buenavista (MHNUC-687), hasta los $2303 \mathrm{msnm}$ en la reserva La Patasola en el municipio de Salento. Seis de los 18 registros (propios y de literatura) se ubican en áreas protegidas de categoría regional, como lo son el Distrito de Conservación de Suelos (DCS) Barbas-Bremen y el Distrito Regional de Manejo Integrado (DRMI) de la Cuenca del río Quindío (Figura 2).

Entre los registros propios se evidenció una variabilidad entre los tipos y el tamaño de los bosques donde se ubican los monos. De los 13 registros propios, la mayoría (seis) se presentaron en bosques cuya vegetación es continua, dos en bosques inmersos en áreas de cultivo y tres más en bosques inmersos en áreas destinadas para potreros. De igual forma se resalta el registro de dos grupos familiares ubicados en dos pequeños fragmentos periurbanos de las ciudades de Armenia (Museo del OroQuimbaya) y Calarcá (Colegio San José). En cuanto a los tamaños de los bosques, siete registros se dieron en bosques de más de 50 hectáreas, tres en bosques entre cinco y 50 hectáreas y tres más en relictos boscosos que no superaron las cinco hectáreas

Mammalogy Notes | Notas Mastozoológicas

Sociedad Colombiana de Mastozoología

Vol. 4 Num. 2| 2018 


\section{Notas | Notes}

(Figura 3). El bosque más extenso donde se registró la presencia de los monos fue el de la reserva La Patasola, el cual se encuentra dentro de un área protegida y cuenta con aproximadamente 130 hectáreas, contrastando con el relicto boscoso del museo del Oro-Quimbaya que cuenta tan solo con 1.5 ha y está inmerso en una matriz urbana.
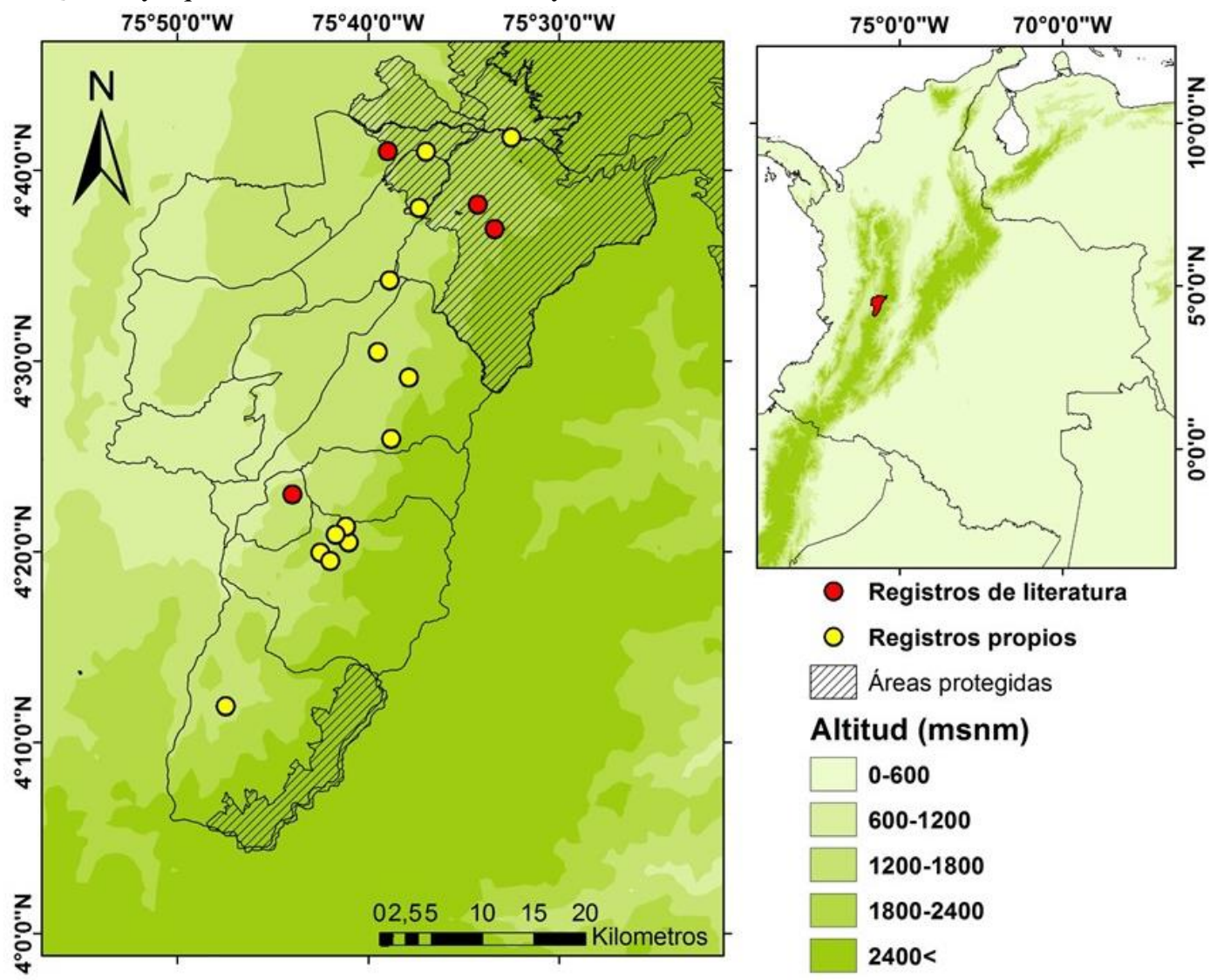

Figura 2. Registros propios y de literatura de A. lemurinus en el departamento del Quindío, Colombia

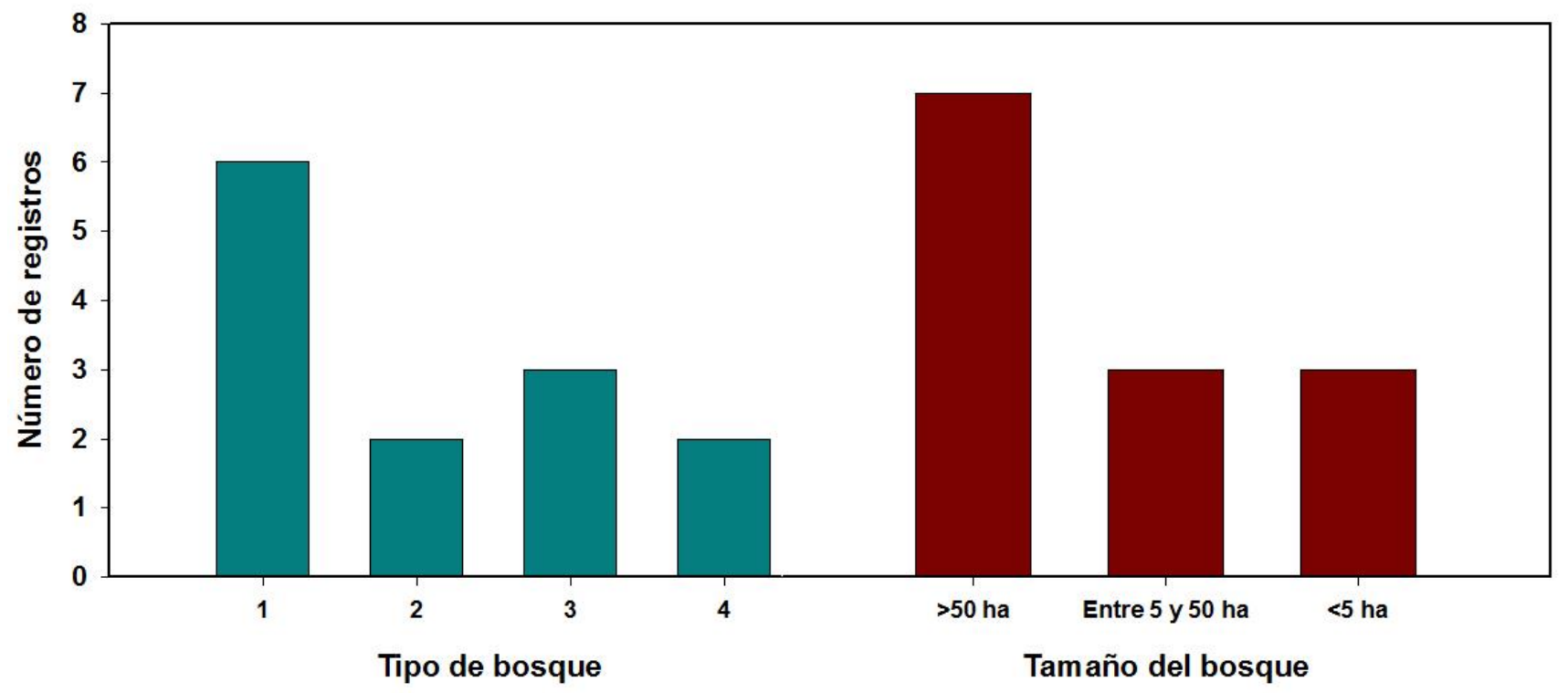

Figura 3. Número de registros de A. lemurinus para cada una de las categorías de tipo de bosque según la composición del paisaje y el tamaño de bosque. Las categorías para el tipo de bosque son: 1. Bosque de vegetación continua; 2; Fragmento de bosque inmerso en cultivos: 3; Fragmento de bosque inmerso en potreros; 4; Fragmento de bosque periurbano.

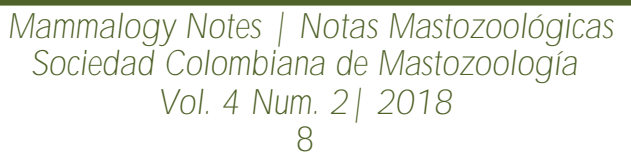




\section{Notas | Notes}

La presencia y distribución de A. lemurinus en el departamento del Quindío ha sido subestimada por los inventarios de mamíferos a nivel nacional (Alberico et al. 2000, Solari et al. 2013), donde indican que esta especie se encuentra en la región de los Andes centrales (Antioquia, Tolima) sin mencionar al departamento del Quindío. Para este departamento, en la actualidad no existe un inventario detallado de mamíferos y los registros publicados de A. lemurinus han sido escasos (GómezHoyos et al. 2014). De igual forma, como lo menciona Castaño \& Cardona (2005), la presencia de los monos nocturnos tiende a ser desapercibida también para la comunidad en general, pues en un 53\% (siete localidades) de las localidades donde se registraron los monos, los habitantes afirmaban que nunca los habían visto.

Tabla 1. Registros propios y de literatura de A. lemurinus en el departamento del Quindío.

\begin{tabular}{|c|c|c|c|c|c|c|c|}
\hline Municipio & Localidad & Coordenadas & $\begin{array}{l}\text { Altitud } \\
\text { (msnm) }\end{array}$ & $\begin{array}{l}\text { Tipo de } \\
\text { registro }\end{array}$ & $\begin{array}{c}\text { Fuente/Número } \\
\text { de colecta }\end{array}$ & $\begin{array}{c}\text { Fecha y hora del } \\
\text { registro }\end{array}$ & $\begin{array}{l}\text { Tipo del Bosque } \\
\text { (área en ha) }\end{array}$ \\
\hline \multirow{5}{*}{ Pijao } & Finca El Billar & $\begin{array}{c}4,33302 \\
-75.70869\end{array}$ & 1709 & Observación & - & $\begin{array}{l}06 / 07 / 2014- \\
01 \mathrm{~h} 40\end{array}$ & $\begin{array}{c}\text { Fragmento de bosque } \\
\text { inmerso en cultivos } \\
(6.58)\end{array}$ \\
\hline & $\begin{array}{l}\text { Vereda Las } \\
\text { Pizarras }\end{array}$ & $\begin{array}{c}4.34166 \\
-75.68388\end{array}$ & 1907 & Observación & - & $\begin{array}{l}\text { 10/10/2016- } \\
\text { 20h15 }\end{array}$ & $\begin{array}{c}\text { Bosque continuo } \\
(67.8)\end{array}$ \\
\hline & $\begin{array}{l}\text { Reserva } \\
\text { Bellavista }\end{array}$ & $\begin{array}{c}4.3255 \\
-75.70011\end{array}$ & 1927 & Observación & - & $\begin{array}{c}11 / 10 / 2016- \\
02 \mathrm{~h} 37\end{array}$ & $\begin{array}{l}\text { Bosque continuo } \\
\qquad(62.4)\end{array}$ \\
\hline & Finca $\mathrm{El}$ bosque & $\begin{array}{c}4.35527 \\
-75.68638\end{array}$ & 2000 & Observación & - & $\begin{array}{l}\text { 17/05/2016- } \\
\text { 00h15 }\end{array}$ & $\begin{array}{c}\text { Bosque continuo } \\
(120.4)\end{array}$ \\
\hline & Finca Mahatma & $\begin{array}{c}4.34833 \\
-75.69527\end{array}$ & 1940 & Observación & - & $\begin{array}{c}\text { 24/08/2015- } \\
19 \mathrm{~h} 50\end{array}$ & $\begin{array}{l}\text { Bosque continuo } \\
(60.3)\end{array}$ \\
\hline Calarcá & Colegio San José & $\begin{array}{c}4.50816 \\
-75.65872\end{array}$ & 1473 & Observación & - & $\begin{array}{c}\text { 21/09/2016- } \\
20 \mathrm{~h} 00\end{array}$ & $\begin{array}{c}\text { Fragmento de bosque } \\
\text { periurbano }(4.2)\end{array}$ \\
\hline Salento & $\begin{array}{l}\text { Reserva La } \\
\text { patasola }\end{array}$ & $\begin{array}{c}4.69530 \\
-75.54208\end{array}$ & 2303 & Observación & - & $\begin{array}{c}13 / 09 / 2014- \\
22 \mathrm{~h} 30\end{array}$ & $\begin{array}{c}\text { Bosque continuo } \\
(130.3)\end{array}$ \\
\hline Filandia & Granja Bengala & $\begin{array}{l}4.68305 \\
-75.61638\end{array}$ & 2200 & Observación & - & $\begin{array}{l}02 / 04 / 2015- \\
21 \mathrm{~h} 50\end{array}$ & $\begin{array}{l}\text { Fragmento de bosque } \\
\text { inmerso en potreros } \\
\text { (75.5) }\end{array}$ \\
\hline Circasia & $\begin{array}{l}\text { Condominio La } \\
\text { Aldea }\end{array}$ & $\begin{array}{c}4.63380 \\
-75.62269\end{array}$ & 1827 & Observación & - & $\begin{array}{c}\text { 18/06/2016- } \\
23 \mathrm{~h} 00\end{array}$ & $\begin{array}{c}\text { Fragmento de bosque } \\
\text { inmerso en potreros } \\
(12.4)\end{array}$ \\
\hline \multirow[b]{2}{*}{ Calarcá } & Vereda el Pensil & $\begin{array}{c}4.43208 \\
-75.64705\end{array}$ & 2014 & Observación & - & $\begin{array}{c}04 / 11 / 2016- \\
20 h 20\end{array}$ & $\begin{array}{c}\text { Bosque continuo } \\
(84.2)\end{array}$ \\
\hline & $\begin{array}{l}\text { Eco-Parque } \\
\text { Peñas Blancas }\end{array}$ & $\begin{array}{c}4.48555 \\
-75.63138\end{array}$ & 1725 & Observación & - & $\begin{array}{l}\text { 25/09/2015- } \\
03 \mathrm{~h} 10\end{array}$ & $\begin{array}{c}\text { Fragmento de bosque } \\
\text { inmerso en potreros } \\
(46.5)\end{array}$ \\
\hline Génova & Río Gris & $\begin{array}{c}4.19861 \\
-75.79138\end{array}$ & 1513 & Observación & - & $\begin{array}{c}\text { 30/03/2016- } \\
\text { 00h30 }\end{array}$ & $\begin{array}{c}\text { Fragmento de bosque } \\
\text { inmerso en cultivos } \\
(4.5)\end{array}$ \\
\hline Armenia & $\begin{array}{l}\text { Museo del Oro- } \\
\text { Quimbaya }\end{array}$ & $\begin{array}{c}4.57055 \\
-75.64833\end{array}$ & 1615 & Observación & - & $\begin{array}{c}15 / 11 / 2016- \\
23 \mathrm{~h} 35\end{array}$ & $\begin{array}{c}\text { Fragmento de bosque } \\
\text { periurbano }(1.5)\end{array}$ \\
\hline Filandia & Vereda Cruces & $\begin{array}{l}4.68333 \\
-75.65\end{array}$ & 1860 & $\begin{array}{c}\text { Observación / } \\
\text { Espécimen de } \\
\text { museo }\end{array}$ & $\begin{array}{c}\text { Castaño \& } \\
\text { Cardona, 2005; } \\
\text { MHNUC-901 }\end{array}$ & $\begin{array}{c}09 / 02 / 2004 \\
05 / 02 / 2004\end{array}$ & - \\
\hline \multirow{2}{*}{ Salento } & - & $\begin{array}{c}4.63680 \\
-75.57113\end{array}$ & 2000 & $\begin{array}{l}\text { Espécimen de } \\
\text { museo }\end{array}$ & $\begin{array}{l}\text { MHNUC- } \\
\text { M00072 }\end{array}$ & $01 / 02 / 1942$ & - \\
\hline & - & $\begin{array}{c}4.61527 \\
-75.55636\end{array}$ & 2134 & $\begin{array}{l}\text { Espécimen de } \\
\text { museo }\end{array}$ & M-33056 & $02 / 10 / 1911$ & - \\
\hline Buenavista & $\begin{array}{l}\text { Finca } \\
\text { Paraguaicito }\end{array}$ & $\begin{array}{c}4.38333 \\
-75.73333\end{array}$ & 1200 & $\begin{array}{l}\text { Espécimen de } \\
\text { museo }\end{array}$ & MHNUC-687 & $17 / 02 / 2006$ & - \\
\hline
\end{tabular}

\footnotetext{
Mammalogy Notes | Notas Mastozoológicas

Sociedad Colombiana de Mastozoología

Vol. 4 Num. 2| 2018
} 
Con respecto a los tipos de bosque donde se ubican los monos nocturnos, varios autores sugieren que A. lemurinus presenta una variación significativa en su uso de hábitat (Castaño \& Cardona 2005, Castaño et al. 2010, Defler 2010). Estos monos pueden llegar a encontrarse tanto en bosques maduros mayores a 2000 ha, hasta zonas altamente transformadas por cultivos y potreros menores a 1,5 ha, e incluso en parches de bosque cercanos a asentamientos humanos (Castaño \& Cardona 2005). Sin embargo, esto último no ha sido confirmado mediante registros exactos, por lo tanto, aquí presentamos los primeros reportes de la presencia del mono nocturno en bosques inmersos en matrices urbanas. El registro de este primate en diferentes bosques del Quindío permite llenar vacíos sobre su distribución en la cordillera central de los Andes. Además, proporciona información, que acompañada de estudios que revelen el efecto de la fragmentación sobre su ecología (Castaño \& Cardona 2005), permitirían generar planes de manejo que promuevan su conservación y reevaluar su categoría de amenaza a nivel nacional.

A partir de los registros aquí reportados, se pretende sensibilizar a la comunidad del Quindío sobre la presencia del Mono Nocturno Andino en diversos bosques del departamento y se recomienda establecer actividades de investigación y educación ambiental, con el fin de generar impactos positivos para la conservación de esta especie de primate.

\section{Agradecimientos}

A Luisa Fernanda Arcila-Pérez y Daniela Gómez-Murcia por información prestada para el registro de los monos en Pijao y Genova. Además, a Héctor E. Ramírez-Chávez, Diego J. Lizcano y Diego Gómez-Hoyos por las revisiones previas al manuscrito.

\section{Referencias}

ALBERICO, M., et al. 2000. Mamíferos (Synapsida: Theria) de Colombia. Biota Colombiana 1:43-75.

ARBELÁEZ-CORTÉS, E., et al. 2011. Birds, Quindío department, Central Andes of Colombia. Check List 7:227-247.

BORRERO, J. I. 1986. La substitución de los cafetales de sombrío por caturrales y su efecto negativo sobre la fauna de vertebrados. Caldasia 15:725-732

CASTAÑO, J. H. \& D. M. CARDONA. 2005. Presencia del mono nocturno andino (Aotus lemurinus I. Geoffroy-St. Hilaire, 1843) en fragmentos de bosque de la cuenca del río Cauca. Boletín Científico del Museo Historia Natural Universidad Caldas 9:111-117.

CASTAÑO, J. H., et al. 2010. Ecología del mono nocturno andino (Aotus lemurinus) en fragmentos de bosque subandino en Colombia. Pp. 69-90 en Primatología en Colombia: Avances al Principio del Milenio (Pereira-Bengoa V, et al. eds.), Fundación Universitaria San Martin, Bogotá, Colombia.

DEFLER, T. R. 2010. Historia Natural de los Primates Colombianos. 2a ed. Universidad Nacional de Colombia, Facultad de Cienci as, Departamento de Biología, Bogotá D.C., Colombia 250-252.

GÓMEZ-HOYOS, D. A., et al. 2014. Representatividad de mamíferos amenazados en el Sistema Departamental de Áreas Protegidas (SIDAP) del Quindío, Colombia. Mammalogy Notes/ Notas Mastozoológicas 1:39-41.

HERNÁNDEZ-CAMACHO, J. I., \& R. W. COOPER. 1976. The nonhuman Primates of Colombia. Pp: 35-69, en: Neotropical Primates: field studies and conservation (Thorington,

J. R \& P. G. Heltne. eds.). National Academy of Sciences, Washington D.C., USA.

MORALES-JIMÉNEZ, A. L. \& S. De La Torre. 2008. Aotus lemurinus. En: IUCN 2008. IUCN Red List of Threatened species. Versión $2016-3$. Downloaded on 16 March 2017. SOLARI, S., et al. 2013. Riqueza, endemismo y conservación de los mamíferos de Colombia. Mastozoología Neotropical 20:301-365. 